\title{
Which B-cell subset should we target in lupus?
}

\author{
Gianfranco Ferraccioli, ${ }^{1}$ Frédéric A Houssiau ${ }^{2}$
}

The role of B-cells in the pathophysiology of lupus nephritis (LN) is suspected since decades. Amazingly, a report from 1977, aimed at describing the pathological characteristics of human LN described 'proliferation of plasma cells (PCs) and plasmacytoid mononuclear cells' in the spleen and the bone marrow (BM), but not in the kidneys. ${ }^{1}$ In murine lupus, Dixon's group discovered that polyclonal B-cell activation was the first detectable immunological abnormality in all strains, which could be observed as early as after 2 weeks of age. ${ }^{2}$ Somewhat later, the same group postulated that murine lupus can be divided into two main types: type 1 murine systemic lupus erythematosus (SLE) (NZB/W and BXSB strains), characterised by primary B-cell hyperresponsiveness to B-cell growth and differentiation factors and type 2 murine SLE (MRL/lpr strain), characterised by T-helper cell hyperactivity and overproduction of B-cell growth factors by proliferating $\mathrm{T}$ cells. ${ }^{3} \mathrm{~A}$ step forward was the discovery by Talal's group that besides polyclonal B-cell activation, a more restricted autoantibody response occurred with time, as suggested by a preferential use of some $\mathrm{VH}$ genes by certain MRL/lpr mice, ${ }^{4}$ thereby leading to further work demonstrating the presence of somatic mutations, affinity maturation and isotype switching as critical mechanisms of pathogenic anti-DNA antibody production in murine and human lupus.

\section{PLASMABLASTS AND PCS}

The production of autoantibodies depends on the capacity of activated and proliferating autoimmune B-cells to become autoantibodysecreting cells (ASCs). During this process, B-cells progressively lose their proliferating ability but increase their autoantibody production. Two types of ASCs have been described: plasmablasts (PBs) and PCs, the former being the precursor of the latter. ${ }^{5} \mathrm{PBs}$

\footnotetext{
${ }^{1}$ Institute of Rheumatology and Affine Sciences, School of Medicine, Catholic University of the Sacred Heart, Rome, Italy; ${ }^{2}$ Rheumatology Department, Cliniques Universitaires Saint-Luc, Université catholique de Louvain, Bruxelles, Belgium

Correspondence to Professor G F Ferraccioli, Institute of Rheumatology and Affine Sciences, School of Medicine, Catholic University of the Sacred Heart, CIC, Via Moscati 31, Rome I-00168, Italy; gf.ferraccioli@rm.unicatt.it
}

are $\mathrm{CD}_{19}{ }^{+} \mathrm{CD} 20^{-} \quad \mathrm{CD}_{2} 7^{+} \mathrm{CD} 38^{++}$ $\mathrm{CD} 138^{ \pm}$highly proliferating IL-6-dependent cells, which have the capacity to migrate. $\mathrm{PCs}$ are $\mathrm{CD} 19^{+} \mathrm{CD} 20^{-} \mathrm{CD} 27^{-} \mathrm{CD} 38^{++}$ $\mathrm{CD} 138^{+}$non-dividing cells, which have lost their migration ability. The transition from B-cells to PBs and PCs is characterised by downregulation of transcription factors $P a x-5$ and Bcl-6 and upregulation of transcription factor Blimp1. Both PBs and PCs are short-lived but, if PCs find a niche in the BM (under normal conditions) or in inflamed tissues (under pathological conditions), they become long-lived PCs. The others die by apoptosis.

In NZB/W mice, PCs can be found in the BM, the spleen and in the kidneys, where they infiltrate the tubulointerstitium of the cortex and the outer medulla. ${ }^{6}$ Interestingly, $40 \%$ of spleen ASC in NZB/W mice are non-dividing long-lived PCs, which cannot be removed by immunosuppressive treatment and continue to produce autoantibodies. ${ }^{7}$ In human lupus, the numbers of circulating PBs and PCs are increased, commensurate with disease activity, as assessed by the Systemic Lupus Erythematosus Disease Activity Index (SLEDAI). ${ }^{8}$ Moreover, PC infiltration was detected in the kidney of patients suffering from more severe renal disease, namely, Class III or IV LN (compared with Class II and V). ${ }^{10}$

\section{KIDNEY STROMAL FACTORS AND PCS FATE}

Stromal cells strongly interact with surrounding infiltrating cells (including those from the B lineage), in particular, by production of several growth factors. Thus, in multiple myeloma (MM), vascular endothelial growth factor, fibroblast growth factor-2 (FGF-2), stromal-derived factor-1 (SDF-1) and hepatocyte growth factor (HGF), all produced by stromal cells, promote $\mathrm{MM}$ cells migration. ${ }^{11}$ HGF is of importance in LN not only as a chemotactic molecule for PCs but also as an antagonist of the profibrotic effects of TGF $\beta 1$ (transforming growth factor $\beta 1$ ). Of interest in human SLE nephritis Peterson et al showed that immunoglobulin heavy (IGHG3) and light (IGL) chains transcripts (B cell signature) marked one of the clusters (cluster IV) and Lyn a Src-family kinase activated after B-cell receptor stimulation also had an increased expression. In addition, B cell transcripts expression correlated significantly with cellular crescents, fibrous crescents and chronicity index, yet TGF $\beta 1$ expression was decreased compared with controls, while no data were provided on HGF expression. ${ }^{12}$ In this respect, the balance between the expression of the two major cytokines, HGF and TGF $\beta 1$, on LN baseline biopsies, was found to predict shortterm renal outcome after treatment with cyclophosphamide. ${ }^{13}$ The counterpart in $\mathrm{NZB} / \mathrm{W}$ mice showed an increased expression of CXCR4 (a chemokine receptor, bindind CXCL12, expressed on PCs) and of Lyn, especially in the interstitium, thus mirroring B-cell findings in human nephritis. ${ }^{14}$ On the whole, these data indicate that the fate and function of PCs in the kidney result from a subtle equilibrium between resident cells, stromal-derived growth factors and the PCs themselves.

\section{TARGETING PCS}

Cheng et $a l^{15}$ used a novel model to demonstrate the role of long-lived PCs in murine LN. They adoptively transferred NZB/W spleen-derived ASCs (a 70/30\% mixture of short-lived PBs/PCs) into $\mathrm{Rag}^{-1-}$ mice (that lack the B-cell lineage) and followed their engraftment in the BM and the kidneys. They demonstrated: (i) that adoptively transferred PBs proliferated in the $\mathrm{BM}$ of $\mathrm{Rag}^{-/}$mice during 2 weeks before becoming non-dividing long-lived PCs; (ii) that these long-lived PCs remained the only surviving transferred cells; (iii) that they continuously produced anti-dsDNA antibodies (more in the BM than in the spleen); (iv) that transferred mice developed immune complex nephritis and had reduced survival; and (v) that treatment with cyclophosphamide (a drug not targeting long-lived non-dividing PCs) was not efficacious in preventing $\mathrm{LN}$. Although some methodological issues can be raised (such as a possible effect of contaminating B1 cells also known for their migratory capacity), the logical conclusion of these experiments is that long-lived PCs should be the target of therapy, a goal currently neither achieved by cytotoxic drugs nor completely by anti-CD20 blockade.

\section{PERSPECTIVES IN HUMAN NEPHRITIS}

Some preliminary evidence suggests that our armamentarium against long-lived PCs may well develop in the near future. First, bortezomib (BTZM), an inhibitor of the $26 \mathrm{~S}$ proteasome, was shown to deplete long-lived PCs in lupus-prone mice by activation of the unfolded protein response and to ameliorate survival of 
such strains (NZB/W and MRL/lpr), ${ }^{16}$ probably through prevention of podocyte damage and loss. ${ }^{17}$ Carfilzomib (another proteasome inhibitor) and BTZM were found to suppress the production of IFNa by TLR-activated plasmacytoid DCs, a purported critical event in the pathogenesis of lupus. ${ }^{18}$ Second, in NZB/W and MRL/lpr mice, a selective inhibitor of Janus Kinase 2 (JAK2), CEP-33779, was recently found to deplete ASCs and longlived PCs defined as CD19-, CD45R/ B2202, CD138hi, CD38hi positive cells, with concomitant reduction of serum IL-12，IL-17A，IFNa，IL-1b and TNFa titres and improved survival. ${ }^{19} 20$ Third, the effects of chronic BLyS/BAFF/APRIL blockade on long-lived PCs are currently unknown (since expression of the corresponding BR3/TACI/BCMA receptors has not been studied) but are not excluded by the fact that CD20 CD138 plasmacytoid cells are progressively depleted by belimumab therapy. ${ }^{21}$ Fourth, Bruton tyrosine kinase inhibitors have been shown to progressively deplete CD138 PCs in the spleen of NZB/W nephritic mice. ${ }^{22}$ Finally, at least from a theoretical viewpoint, Blimp1 pathway inhibitors could be useful.

\section{CONCLUSION}

Type 1-like LN will benefit the most by targeting long-lived PCs, while we have no data on whether targeting $\mathrm{T}$ cells can also lead to wipe out PC in kidney tissues. Targeting of long-lived PCs may become a goal in the clinic, in particular, in LN, where relapses are so common (between $25 \%$ and $35 \%$ of the patients), even years after the first renal insult, ${ }^{23}$ likely because of reactivation of a dormant long-lived PC located in the BM, the spleen or other niches, including the kidneys themselves. In this respect, more attention should be paid to the persistence of residual PCs in renal tissue before and after immunosuppressive treatment, in order not to miss a 'PC window of opportunity'. Toxicity may however be an issue, as targeting of long-lived PCs will likely not be restricted to the autoimmune subset. A strict followup of Ig levels in nephritis patients with low immunoglobulin plasma levels will be necessary.
Funding This paper was partially supported by ASRALES foundation.

Competing interests GFF has received research grants and speakers fee from Roche, UCB, Glaxo, Abbvie, Pfizer, MSD and BMS. FAH has been an investigator or consultant for Aspreva Pharmaceuticals, Bristol Myers Squibb, Human Genome Science/GSK, Merck Serrono, UCB, Astra Zeneka and Roche/ Genentech.

Provenance and peer review Commissioned; externally peer reviewed.

To cite Ferraccioli G, Houssiau FA. Ann Rheum Dis 2013;72:1891-1892.

Received 22 June 2013

Revised 27 July 2013

Accepted 8 September 2013

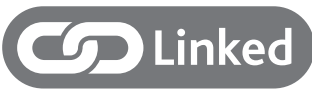

- http://dx.doi.org/10.1136/annrheumdis-2013203455

Ann Rheum Dis 2013;72:1891-1892.

doi:10.1136/annrheumdis-2013-203827

\section{REFERENCES}

1 Fujimoto T, Kitamura T. Pathology of lupus nephritis with special reference to the immunological bases of glomerular changes. Tohotu J Exp Med 1977;122:355-74.

2 Dixon FJ. The pathogenesis of murine systemic lupus erythematosus. Rous - Whipple Award lecture. Am J Pathol 1979:97:10-6.

3 Prud'homme GJ, Fieser TM, Dixon FJ, et al. B-cell tropic interleukins in murine systemic lupus erythematosus (SLE). Immunol Rev 1984;78:159-83.

4 Komisar JL, Leung KY, Crawley RR, et al. Ig VH gene family repertoire of plasma cells derived from lupus-prone MRL//pr and MRL/++ mice. J Immunol 1989:143:340-7.

5 Jourdan M, Caraux A, De Vos J, et al. An in vitro model of differentiation of memory $B$ cells into plasmablasts and plasma cells including detailed phenotypic and molecular characterization. Blood 2009:114:5173-81.

6 Cassese G, Lindenau S, de Boer B, et al. Inflamed kidneys of NZB/W mice are a major site for the homeostasis of plasma cells. Eur I Immunol 2001:31:2726-32

7 Hoyer BF, Moser K, Hauser AE, et al. Short-lived plasmablasts and long-lived plasma cells contribute to chronic humoral autoimmunity in NZB/W mice. J Exp Med 2004:199:1577-84

8 Jacobi AM, Mei H, Hoyer BF, et al. HLA-DRhigh/ CD27high plasmablasts indicate active disease in patients with systemic lupus erythematosus. Ann Rheum Dis 2010;69:305-8.

9 Jacobi AM, Odendahl M, Reiter K, et al. Correlation between circulating CD27high plasma cells and disease activity in patients with systemic lupus erythematosus. Arthritis Rheum 2003;48:1332-42.
10 Espeli M, Bökers S, Giannico G, et al. Local renal autoantibody production in lupus nephritis. J Am Soc Nephrol 2011:22:296-305.

11 Holt RU, Fagerli UM, Baykov V, et al. Hepatocyte growth factor promotes migration of human myeloma cells. Haematologica 2008;93:619-22.

12 Peterson KS, Huang J-F, Zhu J, et al. Characterization of heterogeneity in the molecular pathogenesis of lupus nephritis from transcriptional profiles of laser captured glomeruli. J Clin Invest 2004;113: 1722-33.

13 Capuano A, Costanzi S, Peluso G, et al. Hepatocyte growth factor and transforming growth factor beta1 ratio at baseline can predict early response to cyclophosphamide in systemic lupus erythematosus nephritis. Arthritis Rheum 2006;54: 3633-9.

14 Berthier CC, Bethunaickan R, Gonzales-Rivera T, et al. Cross-species transcriptional network analysis defines shared inflammatory responses in murine and human lupus nephritis. J Immunol 2012;189:988-1001

15 Cheng K, Mumtaz I, Khodadadi L, et al. Autoantibodies from long-lived 'memory' plasma cells of NZB/W mice drive immune complex nephritis. Ann Rheum Dis 2013;72:2011-7.

16 Neubert K, Meister S, Moser K, et al. The proteasome inhibitor bortezomib depletes plasma cells and protects mice with lupus-like disease from nephritis. Nat Med 2008;14:748-55.

17 Hainz N, Thomas S, Neubert K, et al. The proteasome inhibitor bortezomib prevents lupus nephritis in the NZB/W F1 mouse model by preservation of glomerular and tubulointestinal architecture. Nephron Exp Nephrol 2012;120: e47-58.

18 Ichikawa HT, Conley T, Muchamuel T, et al. Beneficial effect of novel proteasome inhibitors in murine lupus via dual inhibition of type I interferon and autoantibody-secreting cells. Arthritis Rheum 2012:64:493-503.

19 Lu LD, Stump KL, Wallace NH, et al. Depletion of autoreactive plasma cells and treatment of lupus nephritis in mice using CEP-33779, a novel, orally active, selective inhibitor of JAK2. J Immunol 2011;187:3840-53.

20 Hainz N, Thomas S, Neubert K, et al. The proteasome inhibitor bortezomib prevents lupus nephritis in the NZB/W F1 mouse model by preservation of glomerular and tubulointerstitial architecture. Nephron Exp Nephrol 2012;120: e47-58

21 Wallace DJ, Stohl W, Furie RA, et al. A phase II, randomized, double-blind, placebo-controlled, dose-ranging study of belimumab in patients with active systemic lupus erythematosus. Arthritis Rheum 2009;61:1168-78.

22 Mina-Osorio P, Lastant J, Keirstead N, et al. Suppression of glomerulonephritis in lupus prone NZB/W mice by RN486, a selective inhibitor of Bruton's Tyrosine Kinase. Arthritis Rheum 2013;65:2380-91.

23 El Hachmi M, Jadoul $M$, Lefèbvre $C$, et al. Relapses of lupus nephritis: incidence, risk factors, serology and impact on outcome. Lupus 2003;12: 692-6. 